\title{
Simplification rationnelle des outils hydrologiques de gestion : recommandations méthodologiques pour la construction de modèles semi-empiriques à origine mécaniste Rational Simplification of Hydrological Mnagement Tools: Methodological Recommandations for Building Semi-Empirical Models of Mechanistic Origin
}

\author{
O. Banton, S. Jordana et M. Larocque
}

Volume 9, numéro 2, 1996

URI : https://id.erudit.org/iderudit/705246ar

DOI : https://doi.org/10.7202/705246ar

\section{Aller au sommaire du numéro}

\section{Éditeur(s)}

Université du Québec - INRS-Eau, Terre et Environnement (INRS-ETE)

\section{ISSN}

0992-7158 (imprimé)

1718-8598 (numérique)

\section{Découvrir la revue}

Citer cet article

Banton, O., Jordana, S. \& Larocque, M. (1996). Simplification rationnelle des outils hydrologiques de gestion : recommandations méthodologiques pour la construction de modèles semi-empiriques à origine mécaniste. Revue des sciences de l'eau / Journal of Water Science, 9(2), 147-161. https://doi.org/10.7202/705246ar

\section{Résumé de l'article}

Les modèles de simulation hydrologiques sont reconnus comme des outils mathématiques très performants mais généralement d'application difficile, principalement à cause du nombre élevé des paramètres requis. À l'inverse, les outils actuels de gestion sont généralement développés à l'aide d'approches empiriques limitant leur application. De plus, leurs paramètres ne sont pas des grandeurs mesurables et doivent être ajustés pour chaque situation. Une approche est proposée pour le développement d'outils semi empiriques de gestion. Elle consiste à simuler un grand nombre de scénarios en utilisant un modèle complexe de simulation puis à rationaliser l'information obtenue pour développer un nouveau modèle semi empirique. L'exemple illustrant cette approche concerne l'évaluation des flux d'eau ruisselée à la surface des champs, lessivée vers la nappe souterraine et drainée par les drains agricoles spécifiquement pour le contexte du Québec. À partir des résultats de simulation de 4500 scénarios, une simplification.rationalisation a permis de réduire à 120 le nombre de scénarios de référence à l'aide desquels peuvent être évalués tous les scénarios possibles par de simples interpolations linéaires. Une application de l'algorithme résultant sur un site du Québec a montré la bonne concordance entre les résultats calculés et mesurés. À la fois l'ordre de grandeur du ruissellement et du drainage et leur grandeur relative sont bien évalués. 


\title{
Simplification rationnelle des outils hydrologiques de gestion : recommandations méthodologiques pour la construction de modèles semi-empiriques à origine mécaniste
}

\author{
Rational simplification of hydrological management tools: \\ Methodological recommandations for building \\ semi-empirical models of mechanistic origin
}

\section{BANTON, S. JORDANA et M. LAROCQUE ${ }^{1}$}

Reçu le 13 octobre 1994, accepté le 13 novembre 1995*.

RÉsuMÉ

Les modèles de simulation hydrologiques sont reconnus comme des outils mathématiques très performants mais généralement d'application difficile, principalement à cause du nombre élevé des paramètres requis. À l'inverse, les outils actuels de gestion sont généralement développés à l'aide d'approches empiriques limitant leur application. De plus, leurs paramètres ne sont pas des grandeurs mesurables et doivent être ajustés pour chaque situation. Une approche est proposée pour le développement d'outils semi-empiriques de gestion. Elle consiste à simuler un grand nombre de scénarios en utilisant un modèle complexe de simulation puis à rationaliser l'information obtenue pour développer un nouveau modèle semi-empirique. L'exemple illustrant cette approche concerne l'évaluation des flux d'eau ruisselée à la surface des champs, lessivée vers la nappe souterraine et drainée par les drains agricoles spécifiquement pour le contexte du Québec. À partir des résultats de simulation de $\mathbf{4 5 0 0}$ scénarios, une simplification-rationalisation a permis de réduire à 120 le nombre de scénarios de référence à l'aide desquels peuvent être évalués tous les scénarios possibles par de simples interpolations linéaires. Une application de l'algorithme résultant sur un site du Québec a montré la bonne concordance entre les résultats calculés et mesurés. $\dot{A}$ la fois l'ordre de grandeur du ruissellement et du drainage et leur grandeur relative sont bien évalués.

Mots-clés : fux, bilan en eau, gestion, modélisation, logiciel.

SUMMARY

Considering the complexity of the water cycie in soil systems, models are used more than ever in parallel with field investigations to assist in the decisionmaking process (KHAKURAL et ROBERT, 1993). Most available models are

1. Institut National de la Recherche Scientifique, Université du Québec, INRS-Eau, 2800 rue Einstein, CP 7500, Sainte-Foy (Québec) Canada. G1V 4C7.

* Les commentaires seront reçus jusqu'au 13 décembre 1996. 
either too complicated (many non-measurable parameters) or too simple (empirical or site-specific) to be used as management tools. Such tools should conform to known theory and should be structured to enable efficient analysis of field situations with minimal requirements for parameters (CARSEL $e t$ al. 1984). However, if the mechanistic models are very performing tools with regards to their representation of the processes and for the accuracy and reliability of their results, they are criticized for their complexity and for the large number of parameters they require. For this reason, their potential application as management tools can not be recommended especially in preliminary investigations when the methodology has to be straight forward and rapidly implemented. On the other hand, existing management tools are often developed using an empirical approach for a specific context which considerably limits their transferability to different situations. Moreover, their empirical parameters often can not be measured for the new situations, and must be adjusted for each new application.

A new approach conciliating the qualities of both kinds of tools was elaborated for the development of management tools. This approach consists in using mechanistic models for simulating a set of possible situations and in rationalizing the information obtained by simulation through regression analyses or other methods. An example of this methodology is presented in this paper with the development of the hydrological part (runoff, leaching and drainage) of a management tool dedicated to the evaluation of nutrient losses related to manure applications. Developed for the Quebec conditions, 4500 theoretical situations were considered corresponding to ten climates, nine soil textures, 25 crops and two slope values. Independently, agricultural management practices and drainage were taken into account.

For the mechanistic simulation of the water budget in the 4500 theoretical situations, the hydrologic module of the mechanistic-stochastic model AgriFlux was used (BANTON et al. 1993b). Because of the important field variability of most parameters, the stochastic AgriFlux model incorporates the variability resulting from field heterogeneity, measurement errors and intrinsic uncertainty related to parameter definition. The soil profile is divided in plot scale homogeneous horizons (or compartments) and a daily time step is used in the calculations. The water budget module in AgriFlux is named HydriFlux and simulates all the water-related processes (precipitations, snowmelt, infiltration, runoff, water uptake by plants, evaporation, percolation and drainage) using characteristic water contents and unsaturated hydraulic conductivity.

In the example presented, the simulation results obtained by running HydriFlux have shown that the soil water fluxes (runoff and percolation) vary as linear functions of both the annual rain volume (the most important characteristic of the climate) and the logarithm of the saturated hydraulic conductivity (the most important characteristic of the soil type). A reduction of the number of crops could also be achieved by taking into account the water needs and the water uptake curves of the crops. This rationalisation-simplification reduced the number of theoretical simulations to be stored in the management tool to 120 ( 2 climates $\times 3$ textures $\times 10$ crops $\times 2$ slopes). These represent only $2.7 \%$ of the initial situations simulated by the mechanistic HydriFlux model. The different water fuxes are stored in the management tool as tables in which direct interpolations are performed to calculate the fluxes corresponding to all the potential intermediary situations. Such developed management tool presents good qualities at the same time for its calculation speed, for its easy parametrisation, for the reliability of its evaluation (through the evaluation of the mechanistic model) and for its high transferability and applicability to various situations. The calculations are rapidly done and their programming can be very easily made by using a spreadsheet software. 
An application of this evaluation method has been done on an experimental site located in Quebec (ENRIGHT et MADRAMOOTOO, 1994), the only one for which both the runoff and the drainage have been measured during many years (1989 to 1991, April to December). The application on two fields (1.84 et $4.63 \mathrm{ha}$ ) has shown a good concordance between the calculated and measured results, as well for the magnitude of the fluxes than for the relative importance of these fluxes. Moreover, this application has shown that the variability of the measured values is higher than the calculated ones, attesting of the great influence of the variations in climatic, soil, crop and management conditions on the water budget. However, the good evaluation of the fiuxes (for relative and absolute values) confirms the reliability of the proposed approach and of the simplification.

Key words : flux, water budget, management, modelling, software.

\section{1 - INTRODUCTION}

Dans le domaine des ressources hydriques et de l'environnement, les outils informatiques de gestion apportent une aide particulièrement précieuse aux gestionnaires. Ils permettent de synthétiser un nombre important d'informations et d'effectuer entre autres le calcul des bilans et l'évaluation des risques potentiels de contamination. Utilisés de pair avec des investigations de terrain et de laboratoire, ils permettent de réaliser rapidement l'étude environnementale de différents systèmes (KHAKURAL et ROBERT, 1993). Pour être utilisés en gestion environnementale, ces outils doivent être basés le plus possible sur la représentation des processus reconnus dans la littérature, représenter le plus efficacement possible la réalité et utiliser le minimum de paramètres (CARSEL et al., 1984). Les logiciels de gestion, conçus pour être facilement utilisés par différents intervenants et gestionnaires des ressources, doivent donc être nécessairement basés sur une représentation fiable mais simplifiée de la réalité. Leurs caractéristiques premières résident alors dans leur souplesse, leur fiabilité et leur facilité d'utilisation dans des contextes multiples et variés.

Dans le domaine de l'hydrologie des sols, de nombreux modèles de gestion ont été développés concernant plus particulièrement l'utilisation des ressources hydriques (JANSSON, 1991), la conservation des sols (WILLIAMS, 1985), le devenir des éléments fertilisants (SHAFFER et al., 1991), celui des pesticides (CARSEL et al., 1984 ; BANTON et al., 1992) et la gestion intégrée de plusieurs de ces composantes (KNISEL, 1980). Ces modèles sont basés sur une approche mathématique détaillée faisant appel à de nombreux paramètres (parfois difficiles à acquérir) ou sur des relations empiriques et globales limitant fortement leur flexibilité et leur applicabilité à différentes situations. Souvent développés pour répondre à des besoins très spécifiques, ces derniers utilisent généralement des constantes empiriques qui ne représentent pas une réalité physique et mesurable, et qu'il est alors nécessaire d'ajuster pour chaque nouveau contexte d'application. Lors de leur développement, de nombreux compromis sont aussi faits dans la représentation des processus pris en compte afin de simplifier l'utilisation et de limiter le nombre de paramètres requis. 
La qualité des outils de gestion réside à la fois dans leur simplicité et dans l'équilibre du compromis réalisé entre la simplification et la fiabilité de la représentation adoptée. II apparaît donc nécessaire de concevoir une approche permettant d'allier les qualités des modèles complexes (représentativité et fiabilité) avec celles des outils plus simplifiés (facilité d'utilisation et de paramétrisation). L'objectif de cette étude est de présenter une nouvelle approche conceptuelle de rationalisation-simplification des outils de simulation hydrologiques complexes pouvant être utilisée dans le développement d'outils de gestion. Cette démarche est illustrée par un exemple d'élaboration de la partie bilan hydrique d'un outil de gestion environnementale de fertilisants organiques (logiciel Fèces ; BANTON et al., 1993a) et par la confrontation des résultats obtenus pour un site expérimental aux valeurs de ruissellement et drainage mesurées.

\section{2 - PROCESSUS DU BILAN HYDRIQUE DU SOL}

Les processus hydriques ayant lieu au niveau du sol sont nombreux. Is concernent principalement le ruissellement et l'érosion de surface, l'infiltration et la percolation dans le sol, l'évaporation et le prélèvement par les plantes, la recharge des nappes et le drainage artificiel souterrain, de même que la dissolution et le lessivage des différents solutés. Leur représentation mathématique est réalisée selon des approches plus ou moins complexes, qui sont choisies et établies en fonction des hypothèses simplificatrices et des critères pris en compte lors du développement des outils.

Parmi ces processus, l'infiltration de l'eau dans le sol et le ruissellement de surface sont ceux qui conditionnent directement l'entrée d'eau au système sol. Ces deux processus du bilan hydrique sont les moins bien connus et les plus difficilement représentables et paramétrisables. Deux approches s'opposent usuellement. La première calcule d'abord le ruissellement selon une approche généralement empirique et très globale. L'infiltration est ensuite calculée comme l'eau précipitée et non ruisselée. Cette approche est principalement utilisée par les outils orientés vers la simulation du ruissellement et de l'érosion, et fonctionnant plutôt à l'échelle du bassin versant (KNISEL, 1980 ; CARSEL et al., 1984), bien que certains logiciels utilisent aussi cette approche à l'échelle de la parcelle. La seconde approche calcule en premier lieu l'infiltration comme le débit d'eau qui peut pénétrer dans le sol et qui est contrôlé par les processus d'humidification et d'écoulement vertical. Le ruissellement est alors le débit d'eau qui ne peut s'infiltrer et qui est contrôlé par la pente et l'état de la surface du sol. Cette approche est généralement utilisée par les outils visant les processus ayant lieu dans le sol et fonctionnant plutót à l'échelle plus fine de la parcelle agricole (BANTON et al., 1993b).

Le mouvement vertical de l'eau dans la partie non saturée du sol est un phénomène relativement bien connu et pour lequel de nombreux algorithmes de simulation ont été développés au cours de la dernière décennie (CARSEL et al., 1984 ; WAGENET et HUTSON, 1989 ; JANSSON, 1991 ; BANTON et al., 1993b). L'écoulement de l'eau dans le milieu non saturé est caractérisé par la présence d'une phase gazeuse influençant les propriétés hydrodynamiques du sol. La loi 
régissant cet écoulement est basée sur une modification de la Loi de Darcy (RICHARDS, 1931). L'équation de Richards utilise la conductivité hydraulique non saturée dont la valeur est une relation directe de la succion matricielle, elle-même fonction du contenu en eau du sol. Selon le type de sol et la connaissance de ses propriétés, la relation mathématique utilisée est plus ou moins fidèle à la réalité du phénomène.

Le devenir de l'eau dans le sol est régi par l'évaporation, la transpiration, l'écoulement de la nappe et le drainage artificiel souterrain. L'évaporation et la transpiration sont calculées en tenant compte de l'eau disponible dans le sol et des valeurs d'évaporation et de transpiration (ou d'évapotranspiration) potentielles qui sont usuellement connues de façon empirique. L'écoulement naturel de la nappe (l'écoulement sub-horizontal de l'eau dans la zone saturée du sol) et le rabattement de celle-ci par les drains souterrains sont régis par la Loi de Darcy appliquée aux écoulements a surface libre. Ce sont des écoulements tridimensionnels à dominante sub-horizontale pour lesquels les représentations mathématiques sont bien connues et maîtrisées. De nombreux modèles en utilisent cependant des représentations simplifiées, surtout quant au nombre de dimensions considérées, entre autres afin de rendre compatibles la structure dimensionnelle des écoulements dans les parties non saturée et saturée du sol.

La discrétisation temporelle, c'est-à-dire le pas de temps du calcul, est un artifice nécessaire à la représentation synthétique des processus. De façon générale, les outils de gestion sont basés sur un pas de temps journatier, sur un pas de temps variable relié à l'apparition de certains événements ou sur un pas de temps annuel. Plus le pas de temps de calcul est petit et s'approche du continuum temporel, plus le modèle est fidèle à la réalité. Le pas de temps journalier permet une représentation assez précise des processus, mais il exige un grand nombre de données d'entrée (climatiques principalement) et peut entraîner un temps de calcul assez long. Le choix d'un intervalle de temps plus long (par événement ou annuel) crée un outil moins exigeant en paramètres, mais aussi nécessairement moins précis au niveau des résultats.

La discrétisation spatiale détermine la position et l'écartement des points de l'espace où ont lieu les calculs. Dans le cas des outils de gestion hydrique des sols, le choix d'une représentation unidimensionnelle verticale s'impose généralement. Plus la discrétisation spatiale est importante, plus les points de l'espace où le bilan est établi sont rapprochés, et plus la représentation adoptée est fidèle à la réalité observable au champ. Toutefois, les calculs impliqués dans une représentation fine sont plus nombreux et plus longs à effectuer, et la discrétisation du sol en quelques couches seulement est usuellement suffisante pour le propos des outils de gestion.

L'ensemble des processus hydriques pris en compte par les modèles génère un système d'équations multiples et complexes dont la résolution devient longue et difficile, particulièrement lorsque les pas de temps et d'espace résultant de la discrétisation sont petits. Les outils les plus performants en terme de représentativité des processus constituent la catégorie des modèles mécanistes (basés sur les mécanismes). Ils requièrent cependant un nombre important de paramètres dont certains sont très difficilement quantifiables ou même évaluables. En conséquence, ces modèles mécanistes performants ne peuvent pas être toujours utilisés comme outils de gestion, surtout lorsque de tels outils doivent répondre à des contraintes pratiques et économiques d'efficacité et d'efficience. 


\section{3- MÉTHODE DE RATIONALISATION-SIMPLIFICATION}

Afin de limiter le plus possible le nombre de paramètres requis par un outil de gestion, tout en exploitant au mieux les principes théoriques utilisés dans les modèles de simulation plus complexes, une approche particulière de simplification et de rationalisation peut être réalisée. Cette approche consiste à appliquer un outil de simulation complexe sur un très grand nombre de cas réels ou théoriques en utilisant les données multiples et détaillées nécessaires à son fonctionnement, puis à exploitér les. résultats ainsi obtenus pour construire un modèle semi-empirique de la même façon qu'avec des données d'observations réelles. Cette approche présente l'avantage de pouvoir prendre en compte un très grand nombre de cas, incluant des scénarios rarement ou difficilement observables dans la réalité, et d'être applicable rapidement sur des contextes pour lesquels peu de résultats réels sont disponibles ou même aucun.

Dans l'outil semi-empirique de gestion Fèces (BANTON et al. 1993a) destiné à l'évaluation sommaire et rapide des pertes potentielles d'azote dans les champs agricoles soumis à l'apport de fumiers et lisiers, le rôle de la partie hydrique est d'évaluer les flux d'eau ruisselée à la surface du champ, lessivée vers la nappe d'eau souterraine et drainée par les drains agricoles souterrains. Cette partie hydrique a été développée en utilisant le module hydrique d'un modèle mécaniste complexe (logiciel Agriflux ; BANTON et al., 1993b) destiné à l'évaluation précise: sur une base journalière des pertes potentielles d'azote. Agriflux comporte, pour la réalisation du bilan en eau, le module Hydriflux qui simule les flux hydriques de surface (infiltration/ruissellement), le flux vertical à travers la zone non saturée du sol (percolation, évaporation, transpiration, lessivage vers la nappe), ainsi que la reprise d'eau par les drains souterrains. Hydriflux est un modèle mécanistestochastique de simulation, c'est-à-dire qu'il est basé sur une représentation conceptuelle des processus (mécanismes) tout en incorporant la variabilité spatiale des paramètres contrôlant ces processus.

Agriflux représente les phénomènes à l'échelle de la parcelle. II permet la définition d'un profil de sol présentant différents horizons ou couches de caractéristiques différentes. Le pas de temps utilisé dans tous les calculs est la journée. Les processus hydriques sont gérés dans les différentes couches du sol (réservoirs) en utilisant une représentation de l'écoulement non saturé basée sur l'équation de RICHARDS (1931) et sur l'approximation de la conductivité hydraulique non saturée par la fonction cubique de IRMAY (1957, citée par BEAR, 1988). Cette fonction, qui ne nécessite que la connaissance des valeurs de capacité au champ et de point de flétrissement, est reconnue comme étant assez fiable pour l'ensemble des sols. À chaque pas de temps (journée), la teneur en eau de chaque couche détermine les processus hydriques pouvant avoir lieu et leur importance respective. Hydriflux prend en compte les précipitations, la fonte de la neige, l'infiltration, le ruissellement, le prélèvement d'eau par la culture, l'évaporation, la percolation, le lessivage vers la nappe souterraine et le drainage artificiel (fig. 1).

L'outil semi-empirique de gestion Fèces a été développé spécifiquement pour le contexte agricole québécois. Des scénarios types ont été sélectionnés puis simulés à l'aide d'Hydriflux, en prenant en compte les conditions hydrologiques les plus fréquentes en milieu agricole. Dix climats (Environnement Canada, 
1984), neuf textures de sol (RAWLS et BRAKENSIEK, 1989) et 25 cultures principales et d'automne (BANTON et al., 1993a) ont été sélectionnés et paramétrisés. Les précipitations annuelles varient de $866 \mathrm{~mm}$ (Saguenay-Lac St-Jean) à $1156 \mathrm{~mm}$ (Québec), les sols d'une conductivité hydraulique de $0,0078 \mathrm{~m} / \mathrm{j}$ (argile) à $7,36 \mathrm{~m} / \mathrm{j}$ (sable) et les cultures d'un besoin potentiel en eau de $0 \mathrm{~mm}$ (pas de culture) à $581 \mathrm{~mm}$ (luzerne). Les combinaisons de toutes ces caractéristiques représentent 4500 situations types (en incluant 2 valeurs de pentes de 0 et $5 \%$ ). Par ailleurs, l'influence du drainage agricole sur le bilan en eau du sol et l'effet des pratiques de travail du sol sont pris en compte. Le nombre d'informations résultant des simulations d'Hydriflux et devant être potentiellement conservées et gérées par l'outil de gestion Fèces est done très élevé et prohibitif pour un outil de gestion. Il est donc nécessaire de limiter les résultats des scénarios tout en conservant l'essentiel de l'information. Cette rationalisation de l'information est réalisée par l'étude des résultats obtenus à l'aide d'HydriFlux.

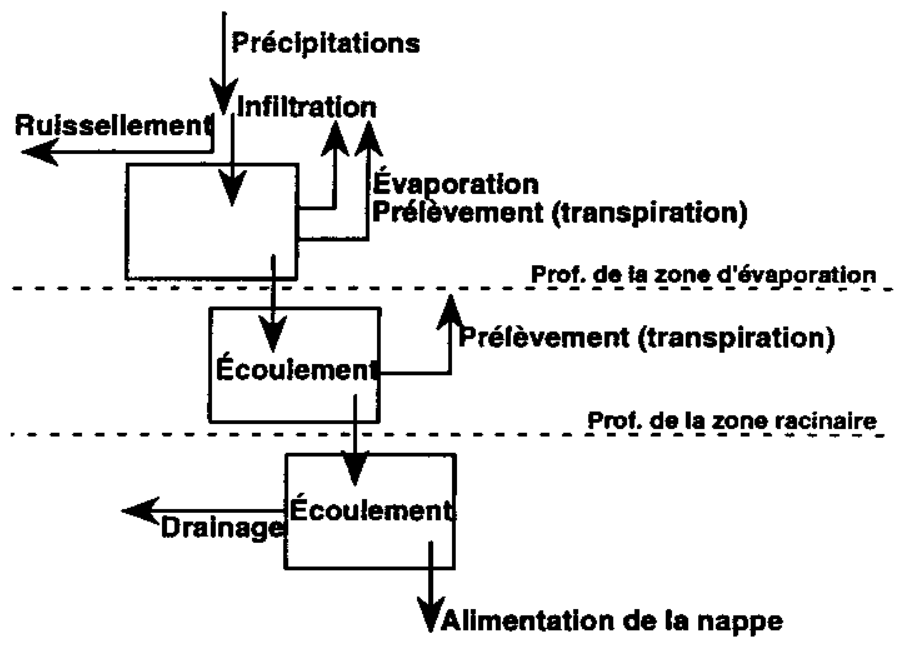

Figure 1 Cycle de l'eau simulé dans Hydriflux.

Water cycle simulated in HydriFlux.

Les simulations montrent que les flux d'eau ruisselés (à la surface du sol) et lessivés (vers la nappe) varient globalement de façon linéaire avec la pluie totale annuelle, pour une paire donnée de texture et culture (fig. 2). L'obtention d'un tel résultat (variation globalement linéaire) est en grande partie attribuable à la relative homogénéité climatique des régions du Québec simulées. Ces droites de régression peuvent être définies à l'aide d'une équation mathématique ou par une interpolation entre deux climats situés aux extrémités de ces droites.

D'autre part, les simulations montrent que les volumes d'eau ruisselée et lessivée sont aussi corrélés avec le logarithme de la conductivité hydraulique saturée, pour un climat et une culture donnés (fig. 3). La conductivité hydraulique saturée est par ailleurs étroitement reliée (log-linéairement) avec la texture du sol (sa composition granulométrique). Les volumes d'eau ruisselée et lessivée 
varient linéairement selon une première droite pour des textures comprises entre l'argile et le loam (limon plus ou moins argileux) et selon une autre droite pour des textures entre le loam et le sable. Les valeurs des flux peuvent donc être interpolées directement entre les flux lessivés et ruisselés de ces trois textures.

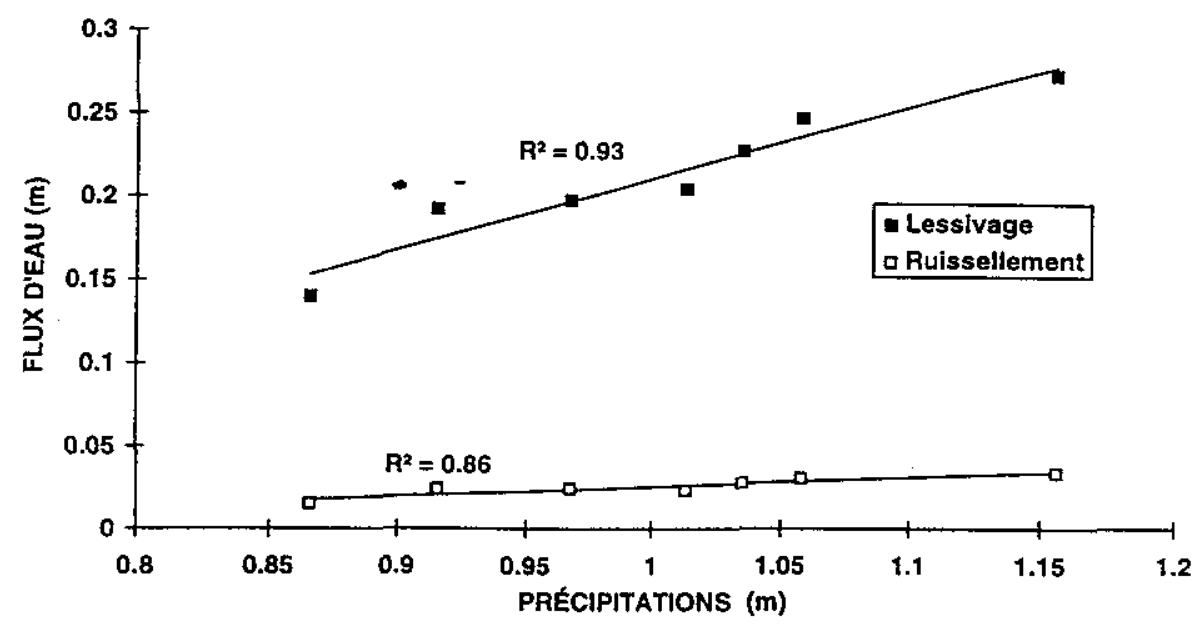

Figure 2 Volumes d'eau ruisselée et lessivée pour différentes précipitations annuelles. Surface and subsurface water flows for various annual precipitations.

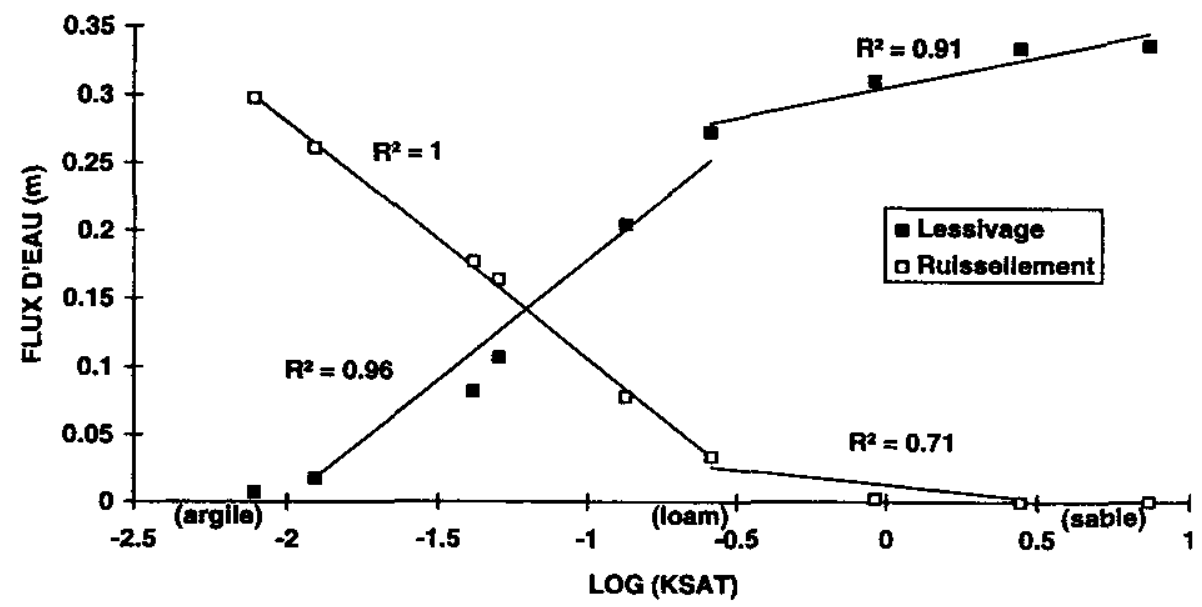

Figure 3 Volume d'eau ruisselée et lessivée pour différentes textures de sol.

Surface and subsurface water flows for various soil textures. 
De plus, plusieurs cultures ont des besoins et des courbes de prélèvement en eau similaires et peuvent donc être regroupées en considérant leur influence sur le bilan hydrique comme identique. Ceci a permis dans notre cas de réduire de 25 à 10 le nombre de cultures types. Toutes les valeurs de flux lessivés et ruisselés pour chacune de ces dix cultures types sont conservées, aucune relation simple n'ayant pu être mise en évidence. Finalement, les simulations ont été.réalisées avec deux conditions de pente $(0$ et $5 \%$ ) et les flux d'eau correspondant à une pente différente sont considérés comme variant linéairement entre ces deux valeurs de pente.

Par ailleurs, les simulations ont permis d'établir une relation entre la texture du sol et la fraction de l'eau infiltrée dañs le sol qui est évacuée par les drains agricoles souterrains (tabl. 1), cette relation étant relativement indépendante des autres facteurs. Finalement, l'importance des travaux de sol a été intégrée de façon complémentaire à la méthodologie de simplification en tenant compte de l'importance du remaniement et de la date de réalisation des travaux. Un système de modification par pondération a ainsi été élaboré (tabl. 2) sur la base des influences reconnues dans la littérature (MWENDERA et FEYEN, 1993; THIBODEAU et MÉNARD, 1993). En effet, le flux lessivé est en général plus important et le flux ruisselé plus faible dans le cas d'un travail du sol augmentant la structure du sol et facilitant donc l'infiltration. Dans l'outil de gestion, les propriétés physiques du sol sont implicitement reliées à sa texture et seule cette dernière caractéristique globale doit être déclarée par l'usager. Ainsi, et selon que la pratique culturale augmente ou diminue la structure du sol et les propriétés qui en dépendent, la texture de base de celui-ci (texture initiale réelle) est décalée vers une texture apparente plus grossière ou plus fine (texture équivalente de calcul).

Tableau 1 Fraction drainée de l'eau infiltrée pour différentes textures de sol.

Table 1 Drained fraction of the infittrated water for various soil textures.

\begin{tabular}{|lc|}
\hline Texture & Fraction drainée \\
\hline Argile & 0,98 \\
Argile limoneuse & 0,97 \\
Argile sableuse & 0,94 \\
Loam argileux & 0,95 \\
Loam limoneux & 0,68 \\
Loam & 0,62 \\
Loam sableux & 0,62 \\
Sable loameux & 0,44 \\
Sable & 0,29 \\
\hline
\end{tabular}

- Résultats obtenus avec un profil de sol comportant deux couches, une première ayant la texture mentionnée et la seconde ayant une texture 100 fois moins permeable. L'interface entre les deux couches correspond à la position des drains.

* Results obtained using a soil profite with two soil compartments, one with the soil texture mentioned and the secondwith a soil texture 100 times less permeable. The drainage tiles are located between the two compartments. 
Tableau 2 Système de pondération de l'influence des travaux de sol.

Table 2 Rating system of the influence of soil management practices.

\begin{tabular}{|ll|}
\hline \multicolumn{1}{|c|}{ Travaux sur le champ } & Points \\
\hline Billoneuse, printemps/été & $2^{*}$ \\
Chisel, printemps & 2 \\
Chisel, automne & 3 \\
Herse à disque, autonomne, un passage & 2 \\
Herse à disque, automne, deux passages & 3 \\
Labour (charrue), automne & 4 \\
Sarcloir, été & 1 \\
Vibroculteur, printemps, un passage & 2 \\
Vibroculteur, printemps, deux passages & 3 \\
\hline
\end{tabular}

* Un total de quatre points n'amène pas de modification de la classe texturale déclarée et de ses propriétés. Une somme inférieure à quatre (présence de résidus végétaux, structure du sol, macropores) favorise f'infiltration (décalage vers une texture plus grossière). Une somme supérieure à quatre favorise le ruissellefaent au détriment de l'infiltration (décalage vers une texture plus fine). Une classe texturale par deux points avec un décalage maximal de deux classes.

* A total of four points corresponds to the user defined soil texture. A total smaller than four favors infitration. A total larger than four increases water runoff. One texture rank by two points with a maximum of two texture ranks.

Cette démarche de simplification-rationalisation permet ainsi de réduire à 120 ( 2 climats $\times 3$ textures $\times 10$ cultures $\times 2$ pentes) le nombre de scénarios de base simulés avec Hydriflux dont l'information est conservée dans l'outil de gestion Fèces. Cette information, c'est-à-dire les flux d'eau lessivée et ruisselée pour chacun des scénarios, est stockée sous forme de tableaux (tabl. 3), à l'intérieur desquels sont effectuées de simples interpolations pour l'évaluation des flux spécifiques à un quelconque scénario. Les informations demandées par l'outil développé pour le bilan en eau se limitent au choix de l'un des dix climats proposés, de l'une des neufs textures de sol et de l'une des 25 cultures combinées, à la déclaration de la pente du sol, à la déclaration d'un système de drainage et à l'identification des travaux de sol parmi une liste de travaux potentiels. Cette information est donc très simple et constitue dans tous les cas l'information minimale toujours connue pour le scénario hydro-pédologique à évaluer. Une telle simplicité de l'information requise convient bien à un outil de gestion visant entre autres une évaluation rapide de scénarios pour lesquels l'information détaillée n'est pas toujours disponible ou facile à obtenir. Malgré le peu d'informations demandées, les flux annuels d'eau ruisselée, drainée et lessivée fournis par l'outil de gestion sont le résultat de simulations complexes effectuées en prenant en compte plus d'une dizaine de processus (représentés par des algorithmes mathématiques complexes) et faisant appel à plus d'une trentaine de paramètres physiques différents (correspondant à des réalités mesurables). L'exemple présenté montre le gain important réalisable au niveau de la simplification de l'information nécessaire pour l'utilisation de l'outil, tout en conservant les qualités globales des outils de simulation plus complexes pour l'obtention de résultats fiables. 
Tableau 3 Exemples de matrices pour l'évaluation des flux ruisselé et drainé par interpolation.

Table 3 Examples of the matrix to evaluate the drained and leached water fluxes by interpolation.

\begin{tabular}{|c|c|c|c|c|c|}
\hline \multicolumn{2}{|c|}{ Arglle * } & \multicolumn{2}{|c|}{ Loam } & \multicolumn{2}{|c|}{ Sable } \\
\hline \multicolumn{6}{|c|}{ RUISSELLEMENT SOUS CULTURE DU MAĨS } \\
\hline $\begin{array}{c}0 \\
168\end{array}$ & $\begin{array}{c}0 \\
298\end{array}$ & $\begin{array}{c}0 \\
18\end{array}$ & $\begin{array}{c}0 \\
33\end{array}$ & $\begin{array}{l}0 \\
0\end{array}$ & $\begin{array}{l}0 \\
0\end{array}$ \\
\hline \multicolumn{6}{|c|}{ LESSIVAGE SOUS CULTURE DU MAÏS } \\
\hline $\begin{array}{c}29 \\
4\end{array}$ & $\begin{array}{c}82 \\
7\end{array}$ & $\begin{array}{l}172 \\
158\end{array}$ & $\begin{array}{l}304 \\
275\end{array}$ & $\begin{array}{l}204 \\
204\end{array}$ & $\begin{array}{l}338 \\
338\end{array}$ \\
\hline \multicolumn{6}{|c|}{ RUISSELLEMENT SOUS CULTURE DU MIL ** } \\
\hline $\begin{array}{c}0 \\
203\end{array}$ & $\begin{array}{c}0 \\
429\end{array}$ & $\begin{array}{c}0 \\
19\end{array}$ & $\begin{array}{c}0 \\
38\end{array}$ & $\begin{array}{l}0 \\
0\end{array}$ & $\begin{array}{l}0 \\
0\end{array}$ \\
\hline \multicolumn{6}{|c|}{ LESSIVAGE SOUS CULTURE DU MIL * * } \\
\hline $\begin{array}{c}83 \\
9\end{array}$ & $\begin{array}{l}83 \\
19\end{array}$ & $\begin{array}{l}223 \\
205\end{array}$ & $\begin{array}{l}448 \\
411\end{array}$ & $\begin{array}{l}248 \\
248\end{array}$ & $\begin{array}{l}473 \\
473\end{array}$ \\
\hline \multicolumn{6}{|c|}{ RUISSELLEMENT SANS CULTURE } \\
\hline $\begin{array}{c}0 \\
173\end{array}$ & $\stackrel{0}{350}$ & $\begin{array}{c}0 \\
18\end{array}$ & $\begin{array}{c}0 \\
35\end{array}$ & $\begin{array}{l}0 \\
0\end{array}$ & $\begin{array}{l}0 \\
0\end{array}$ \\
\hline \multicolumn{6}{|c|}{ LESSIVAGE SANS CULTURE } \\
\hline $\begin{array}{c}69 \\
4\end{array}$ & $\begin{array}{l}83 \\
12\end{array}$ & $\begin{array}{l}178 \\
163\end{array}$ & $\begin{array}{l}360 \\
327\end{array}$ & $\begin{array}{l}209 \\
209\end{array}$ & $\begin{array}{l}388 \\
388\end{array}$ \\
\hline
\end{tabular}

- Les quatre valeurs indiquées pour chaque texture correspondent à gauche à la précipitation minimale ( $860 \mathrm{~mm}$ ), à droite à la précipitation maximale $(1160 \mathrm{~mm})$, en haut a la pente nulle et en bas a la pente de $5 \%$. Les flux sont interpolés entre ces quatre póles.

* * Le ruissellement et le lessivage sont plus importants pour la culture de mil que pour un sol nu du fait d'une couche résiduelle de neige plus importante (maintenue par les tiges de mil) et d'un besoin en eau faible du mil.

* The four values indicated for each grain size correspond at left to the minimum rain fevel $(860 \mathrm{~mm})$, at right to the maximum one $(1160 \mathrm{~mm})$, at the top to the slope $0 \%$ and at the bottom to the slope $5 \%$. The fluxes are interpolated between these four poles.

- Runoff and leaching of water are higher for the millet crop than for the nude soll surface because of the higher snow cover staying on the soil (retained by the stems) and the lower water need of the crop.

\section{4 - APPLICATION AU BILAN HYDRIQUE D'UN SITE EXPÉRIMENTAL}

Bien qu'un tel outil ne permette pas l'étude précise d'une situation réelle, il est cependant pertinent d'en faire une application sur un contexte connu pour en illustrer la validité et la limitation. Le site expérimental utilisé pour cette illustration est le seul site agricole du Québec pour lequel une information est disponible concernant le ruissellement et le drainage. Sur ce site localisé à St-Léonardd'Aston (région de Nicolet, Québec, Canada), une étude a été réalisée de 1989 à 
1991 (ENRIGHT et MADRAMOOTOO, 1994). Elle a consisté à mesurer durant les périodes d'avril à décembre le ruissellement de surface et le drainage à l'aide de déversoirs sur deux parcelles dont les tailles respectives sont 1,84 et 4,63 ha. Le bilan hydrique n'est cependant pas complet puisque d'une part les périodes suivies (courant avril à courant décembre) ne couvrent pas toute l'année hydrologique même si le gel hivernal limite les flux d'eau, et que d'autre part ni l'écoulement naturel de la nappe ni l'évaporation ne sont mesurés.

Sur ces deux parcelles, respectivement constituées d'un sable loameux et d'un loam sableux (série St Jude), la culture principale est la pomme de terre (en 1989 et 1990) cultivée en rotation de 2 à 3 ans avec de l'orge et du trèfle (en 1991). Du seigle d'hiver a de ptus été semé à l'automne 1990 sur le second site. Le premier site présente une pente longitudinale de $0,7 \%$ avec présence de billons ayant une pente latérale de $2,9 \%$ (la pente moyenne résultante considérée est de $1,53 \%$ ) alors que le second site présente une pente uniforme de $0,73 \%$. Les travaux de sol sont conventionnels (labour d'automne et hersage de printemps) avec un travail réduit durant l'année de céréale (1991). Les mesures de conductivité hydraulique réalisées par les chercheurs ont mis en évidence une compaction importante du sol sur les passages de roues entraînant une conductivité hydraulique 11,5 fois plus faible dans ces secteurs et affectant plus de $25 \%$ de la surface des champs. Considérant cela, les calculs ont entrainé selon la méthode employée par l'outil un décalage des sols des deux parcelles de deux classes texturales (une classe texturale pour l'effet destructurant du travail du sol, l'autre pour l'effet local de la compaction). Les deux sites sont équipés d'un réseau de drainage souterrain installé à une profondeur de 1,2 m.

Les résultats obtenus par la méthode développée sont présentés au tableau 4 avec les mesures aux champs. L'analyse des résultats fait ressortir d'une part que l'année 1990 se différencie fortement des autres (au niveau des valeurs mesurées) et d'autre part que les grandeurs de ruissellement et de drainage sont globalement bien évaluées. En effet, les valeurs de ruissellement pour 1989 et 1991 varient entre 1,6 et $51,2 \mathrm{~mm}$ pour les mesures et entre 7,7 et $10,7 \mathrm{~mm}$ pour les calculs, alors que les valeurs de drainage varient entre 87,0 et $222,8 \mathrm{~mm}$ pour les mesures et entre 134,3 et 154,4 mm pour les calculs. Ces résultats font ressortir la grande variabilité des valeurs mesurées $( \pm 94 \%$ et $\pm 44 \%$ respectivement pour le ruissellement et le drainage) devant celle des valeurs calculées (respectivement $\pm 16 \%$ et $\pm 7 \%$ ). Ceci était cependant attendu puisque l'évaluation calculée correspond à des conditions moyennes de climat, sol et culture alors que les valeurs mesurées sont fortement influencées par les différentes variations. Le facteur influençant le plus considérablement les résultats ponctuels est la variation temporelle des précipitations. Tel que le montrent les valeurs mesurées, les précipitations de 1990 (avec 736,4 mm durant la saison d'observation) ont entraîné des flux de drainage deux fois plus élevés sur les deux sites que les années 1989 et 1991 (628,5 et $652,0 \mathrm{~mm}$ respectivement pour la même période). L'année pluvieuse de 1990 se différencie donc fortement des évaluations réalisées par le calcul. La régression linéaire réalisée entre les flux mesurés et évalués présente une pente de 0,37 et un coefficient de corrélation $(r)$ de 0,72 lorsque 1990 est pris en compte, alors que la pente est de 0,82 et le coefficient de corrélation de 0,87 lorsque 1990 est écarté. L'importance relative du ruissellement et du drainage, qui constituent les exportations hors des champs, est cependant toujours bien évaluée dans les deux cas. Ainsi, la régression linéaire entre les grandeurs relatives du ruissellement et du drainage pour les deux sites 
et les trois années donne une pente de 1,04 et un coefficient de corrélation de 0,95 confirmant la bonne évaluation des grandeurs relatives des deux phénomènes. De plus, on peut considérer que l'ordre de grandeur du ruissellement et du drainage est bien obtenu par la méthode puisque les valeurs de ruissellement qui peuvent être fournies par le calcul pour toute la gamme possible de sols, climats, pentes et cultures varient de 0 à $429 \mathrm{~mm}$ alors que celles du drainage varient de 2 à $473 \mathrm{~mm}$. La comparaison des résultats calculés aux valeurs mesurées montre donc la bonne qualité des évaluations réalisées dans ce cas d'application. L'application de cet algorithme de calcul, et à travers lui de la méthode de simplification proposée, peut donc être envisagée avec confiance pour l'évaluation sommaire et préliminaire des flux annuels de ruissellement et de drainage sur les champs, tant au niveau de leur grandeur relative qu'absolue.

Tableau 4 Comparaison des flux mesurés et calculés.

Table 4 Comparison of the measured and calculated fluxes.

\begin{tabular}{|cccccc|}
\hline Parcelle & Année & \multicolumn{2}{c}{ Ruissellement $(\mathrm{mm})$} & \multicolumn{2}{c|}{ Drainage (mm) } \\
\hline & & Mesuré & Simulé & Mesuré & SImulé \\
\hline 1 & 1989 & 1.6 & 7.7 & 137.3 & 143.6 \\
1 & 1990 & 6.1 & 7.7 & 466.2 & 143.6 \\
1 & 1991 & 1.6 & 7.8 & 222.8 & 154.4 \\
2 & 1989 & 51.2 & 10.7 & 87.0 & 134.3 \\
2 & 1990 & 43.7 & 9.8 & 187.6 & 128.4 \\
2 & 1991 & 15.5 & 10.5 & 106.9 & 145.2 \\
\hline
\end{tabular}

\section{5 - CONCLUSION}

Une nouvelle approche de rationalisation-simplification des outils complexes de simulation pouvant être utilisée pour le développement d'outils de gestion plus simples a été présentée dans cet article. Cette nouvelle approche allie les principales qualités des modèles mécanistes de simulation (fiabilité) et des outils de gestion classiques (simplicité). Elle utilise d'une part les modèles mécanistes pour simuler un ensemble de scénarios probables et d'autre part une rationalisation de l'information obtenue de ces simulations principalement par le biais d'études de régression.

Un exemple a été présenté, comme illustration de la démarche proposée, pour le développement de la partie hydrique d'un outil de gestion des pertes environnementales de fertilisants organiques agricoles (logiciel Fèces). Ce développement, réalisé pour les conditions québécoises, a nécessité la simulation de 
4500 scénarios types concernant dix climats, neuf textures de sol, 25 cultures combinées et deux valeurs de pente à l'aide d'un modèle mécaniste-stochastique (module Hydriflux du logiciel Agriflux). Par ailleurs et indépendamment, l'influence du drainage et celle du travail du sol ont été prises en compte. Les résultats obtenus de ces simulations ont permis d'identifier les possibilités de simplification et de rationalisation puisque cet exercice a permis de réduire à 120 le nombre de scénarios de base simulés avec HydriFlux dont l'information est conservée dans l'outil de gestion Fèces. Ce sont donc les résultats de seulement $2,7 \%$ des scénarios initiaux (simulés par HydriFlux) qui sont conservés dans l'outil développé.

Les calculs résultant de cette démarche étant très rapidement effectués et très facilement programmables, un tel outil peut être facilement développé et implanté sur un simple chiffrier informatique (tableur). Un outil de gestion ainsi développé présente à la fois les qualités de rapidité de calcul, de paramétrisation simple, de fiabilité de l'évaluation (héritée du modèle mécaniste) et de transférabilité à de nombreuses situations existantes ou probables. L'application de l'algorithme résultant sur un site expérimental du Québec a montré une bonne concordance entre les résultats calculés et ceux mesurés aux champs. En effet, à la fois l'ordre de grandeur du ruissellement et du drainage et leur grandeur relative sont bien évalués. Cette application a par ailleurs montré que la variabilité des valeurs mesurées est plus élevée que celle des calculs, attestant de l'influence des variations climatiques, pédologiques et culturales sur le bilan hydrique d'un sol. Cependant, la bonne évaluation des flux (de façon relative et absolue) confirme la validité de l'approche utilisée et des choix de simplification réalisés.

\section{REMERCIEMENTS}

Les auteurs remercient le Ministère de l'Environnement et de la Faune du Québec pour leur contribution financière, ainsi que le Gouvernement Espagnol pour l'octroi d'une bourse de recherche à M. Jordana.

\section{RÉFÉRENCES BIBLIOGRAPHIQUES}

BANTON O., JORDANA S., LAROCQUE M., 1993a. Féces. Bilan environnemental des fertilisants organiques. Manuel de l'utilisateur. INRS-Eau. Ste-Foy, Québec, Canada.

BANTON O., LAROCQUE M., SURATEAU F., VILLENEUVE J.-P., 1993b. AgriFlux: Logiciel d'évaluation des pertes environnementales de contaminants agricoles.
Manuel d'usager. Rapport d'étape $n^{\circ} 3$. Rapport scientifique $n^{\circ} R-380$, INRSEau. Ste-Foy, Québec, Canada.

BANTON O., LAFRANCE P., MARTEL R., VILLENEUVE J.P., 1992. Planning of soilpore water sampling campaigns using pesticide transport modelling. Ground Water Monitoring Review. 12 (3) : 195202. 
BEAR J. 1988. Dynamics of fluids in porous media. Dover Publications Inc. New York, NY.

CARSEL R.F., SMITH C.N., MULKEY L.A., DEAN J.D., JOWISE P., 1984. User's manual for the pesticide root zone model (PRZM). U.S. EPA. Athens, GA.

ENRIGHT P., MADRAMOOTOO C.A., 1994. Hydrologic response of surface and subsurface drained agricultural fields. Can. Agric. Eng. 36 (1) : 15-24.

ENVIRONNEMENT CANADA, 1984. Nörmales climatiques au Canada, 1951-1980. Service de l'environinement atmosphérique. Publication du programme climatologique canadien.

JANSSON P.-E., 1991. Simulation model for soil water and heat conditions. Description of the SOIL model. Swedish Univ. of Agric. Sciences. Department of Soil Sciences, Uppsala, Sweden.

KHAKURAL B.R., ROBERT P.C., 1993. Soil nitrate leaching potential indices using a simulation model as a screening system. J. Environ. Qual. 22 : 839-845.

KNISEL W.G., 1980. CREAMS : A field-scale model for chemicals, runoff and erosion from agricultural management systems. Conservation Research report 26. U.S. Dept. Agric. Washington D.C.

MWENDERA E.J., FEYEN J., 1993. Predicting tillage effects on infiltration. Soil Sci. $155: 229-235$
RAWLS W.J., BRAKENSIEK D.L., 1989. Estimation of soil water retention and hydraulic properties. Dans: Unsaturated flow in hydrologic modeling theory and practice. H.J. Morel-Seytoux (ed.). Kluwer Academic Publ. Pays-Bas.

RICHARDS L.A., 1931. Capillary conduction of liquids in porous mediums. Physics. 1 : 318-333.

SHAFFER M.J., HALVORSON A.D., PIERCE F.J., 1991. Nitrate leaching and economic analysis package (NLEAP): model description and application. In: Follet et al. (Eds) Managing Nitrogen for Groundwater Quality and Farm Profitability. Soil Sci. Soc. A., Inc. Madison, WI.

THIBODEAU S., MÉNARD O., 1993. Pratiques agricoles de conservation; ce qu'elles sont, ce qu'elles font, ce qu'elles valent. Colloque sur la gestion de l'eau, CPVQ. 20-21 avril 1993. St-Hyacinthe, Canada.

WAGENET R.J., HUTSON J.L., 1989. LEACHM : Leaching Estimation and Chemistry Model : A process based model of water and solute movement transformation, plant uptake and chemical reaction in the unsaturated zone. Water Resources Institute, Cornell University. Ithaca, NY.

WILLIAMS J.R., 1985. The physical components of the EPIC model. Soil erosion and conservation. El Swaify, Moldenhauer and Andrew (eds). Ankeny, lowa. Soil Conservation Society of America, pp. 272-284. 